\title{
EARLY FETAL HYDROPIC CHANGES ARE ASSOCIATED WITH MODERATE DILATATION OF THE BRAIN VENTRICULAR SYSTEM: A CLUE TO A POSSIBLE LINK BETWEEN CERVICAL LYMPHATIC ENGORGEMIENT AND VENTRICULAR DILATATION?
}

D. Paladini, G. Donarini, A. Conti, L. Costanza De Angelis, M.H. Witte, R. Genesio, M. Bernas, T. Bellini, F. Boccardo, L.A. Ramenghi, C. Bellini

Fetal Medicine \& Surgery Unit (DP,GD), Gaslini Institute, Genoa, Italy; Cytogenetics (AC), University Federico II of Naples, Italy; Dept. of Mother \& Child (LCDA,LAR,CB), Neonatal Intensive Care Unit, Gaslini Institute, Genova, Italy; Dept. of Surgery (MHW), University of Arizona College of Medicine, Tucson, AZ; Dept. of Medical Education (MB), TCU and UNTHSC School of Medicine, Fort Worth, TX, USA; and Dept. of Surgery (FB), University of Genova, Italy

\section{ABSTRACT}

The aim of this study is to assess whether early cervical lymphatic obstruction is associated with a sonographically detectable dilatation of the ventricular system in the 1st trimester of pregnancy. In particular, the objective is to assess whether fetuses with non-immune hydrops fetalis (NIHF), cystic hygroma, or enlarged nuchal translucency (NT) have a greater atrial width/biparietal diameter (AW/BPD) ratio than normal at time of the combined first trimester screening scan. This retrospective study included 96 first trimester fetuses (33 normal and 63 with various degree of cervical lymphatic engorgement). Inclusion criteria were CRL in the 45-84 $\mathrm{mm}$ range and availability of one or more three-dimensional volume datasets of the fetal head, acquired from the BPD plane. Each three-dimensional volume dataset was opened and multiplanar correlation employed to align the three orthogonal planes. The ratio between the atrial width and the BPD (AWIBPD ratio) was used to evaluate the possible presence of increased amount of cerebrospinal fluid. Abnormal cases were placed into 4 categories: 1) enlarged non-septated NT $2.5-3.9 \mathrm{~mm}$, no hydrops; 2) grossly enlarged non-septated NT
/ edema $>3.9 \mathrm{~mm}$; 3) cystic hygroma and/ or NIHF; 4) major anomalies with $N T<2.5$ $\mathrm{mm}$. Presence of dilatation of the laterocervical jugular lymphatic sacs, karyotype and presence of congenital anomalies were also recorded. The One-way ANOVA test was used to compare means. Intra- and inter-observer variability were also assessed. The AWIBPD ratio was found to be significantly higher in fetuses with grossly enlarged NT/nuchal edema and NIHF/septated cystic hygroma than in normal $(p<0.05$ and $p<0.01$, respectively). Also, the AW/BPD ratio was significantly higher in NIHF/septated cystic hygroma than in enlarged NT $2.5-3.9 \mathrm{~mm}(p<0.05)$. In case of enlarged NT (2.5-3.9 mm), the AW/BPD ratio is significantly higher in presence of JLS $(p<0.01)$. At the end of the first trimester, presence of cervical lymphatic engorgement, in terms of grossly enlarged NT, nuchal edema, septated cystic hygroma, and NIHF, is statistically associated with a moderate dilatation of the ventricular system. Of note, among fetuses with moderately enlarged NT, those with evidence of dilatation of the JLS show a statistically significant increase in the AWIBPD ratio. 
Keywords: fetus, hydrops, cystic hygroma, nuchal translucency, brain lymphatic, cerebrospinal fluid, ultrasound

Non-immune hydrops fetalis (NIHF) is a disease still far from being thoroughly understood $(1,2)$. The pathogenesis of primary lymphatic dysplasia and that of hydropic changes secondary to cardiovascular or other causes are partly overlapping and a defined understanding of the normal and abnormal development of the lymphatic system is still lacking. In brief, since the brain is completely devoid of lymphatics, it seems that both cerebrospinal and interstitial fluid convey waste and solutes, with complex mechanisms, across the cribriform plate, to the cervical lymphatics (3). It is possible to speculate that, in the presence of overt NIHF and/or cystic hygroma or thickened Nuchal Translucency, a delayed or abnormal development of the fetal cervical lymphatic network may determine a reduction in the drainage of (cerebrospinal fluid) CSF via the perineural space through the cribiform plate. The hypothesis of this study is if this mechanism is indeed active in the human fetus, then in those cases in which an engulfment of the primary lymphatic system is present, e.g., NIHF, cystic hygroma, and enlarged nuchal translucency, there should be an increase in the amount of the cerebrospinal fluid in the fetal brain. To assess this hypothesis, we measured width of the lateral ventricle measured on an axial view of the fetal head at the level where the choroid plexuses are more prominent. By normalizing then the atrial width (AW) by the biparietal diameter BPD (AW/BPD), we accounted for the gestational age changes. The aim of this study is to assess whether early cervical lymphatic obstruction is associated with a sonographically detectable dilatation of the ventricular system in the 1st trimester of pregnancy. In particular, the objective is to assess whether fetuses with NIHF, cystic hygroma or enlarged nuchal translucency have a greater AW/BPD ratio than normal at the time of the combined first trimester screening scan.

\section{METHODS}

This is a retrospective study conducted on a series of 33 normal fetuses and 63 fetuses with enlarged NT, and/or chromosomal and/ or congenital anomalies evaluated at two tertiary referral centers (Fetal Medicine \& Surgery Unit, Giannina Gaslini Institute, Genoa, Italy, and Fetal Medicine \& Cardiology Unit, Dept of Obstetrics and Gynecology, University Federico II of Naples, Italy) over 5 years. All fetuses had undergone ultrasound aiming at the measurement of the Nuchal Translucency (NT) and the visualization of the Nasal Bone according to the recommendations of the Fetal Medicine Foundation (4). Inclusion criteria were crown-rump length (CRL) in of 45-84 mm and availability of one or more three-dimensional volume datasets of the fetal head, acquired from the BPD plane. Twenty-one cases with NT enlargement had to be excluded because only volumes acquired from the midsagittal view of the fetal head were available. The technique to measure the AW/BPD ratio is the following. Each volume dataset was accessed with a dedicated software (4D viewer, version 9.0, GE medical system, Milwaukee, IL). Once the volume of the fetal head was opened, the axial view appearing on the A panel was aligned on the three orthogonal planes, using the reference dot on multiplanar correlation imaging. In this way, we were certain that the axial plane was not erroneously tilted on one side. The image on the A plane was then magnified and the calipers positioned on the echoic lines representing the walls of the atrium at the level of the choroid plexus. The atrial width measurement was taken with or without the addition of Volume Contrast Imaging with $1 \mathrm{~mm}$ thick slices, according to whether the actual edges of the lateral ventricle were seen more or less reliably. Care was taken not to include in the measurement part of the developing brain parenchyma. In case of doubt, if the choroid plexus touched the walls of the atrium, multiplanar image correlation was employed to avoid measuring errors with checking the 


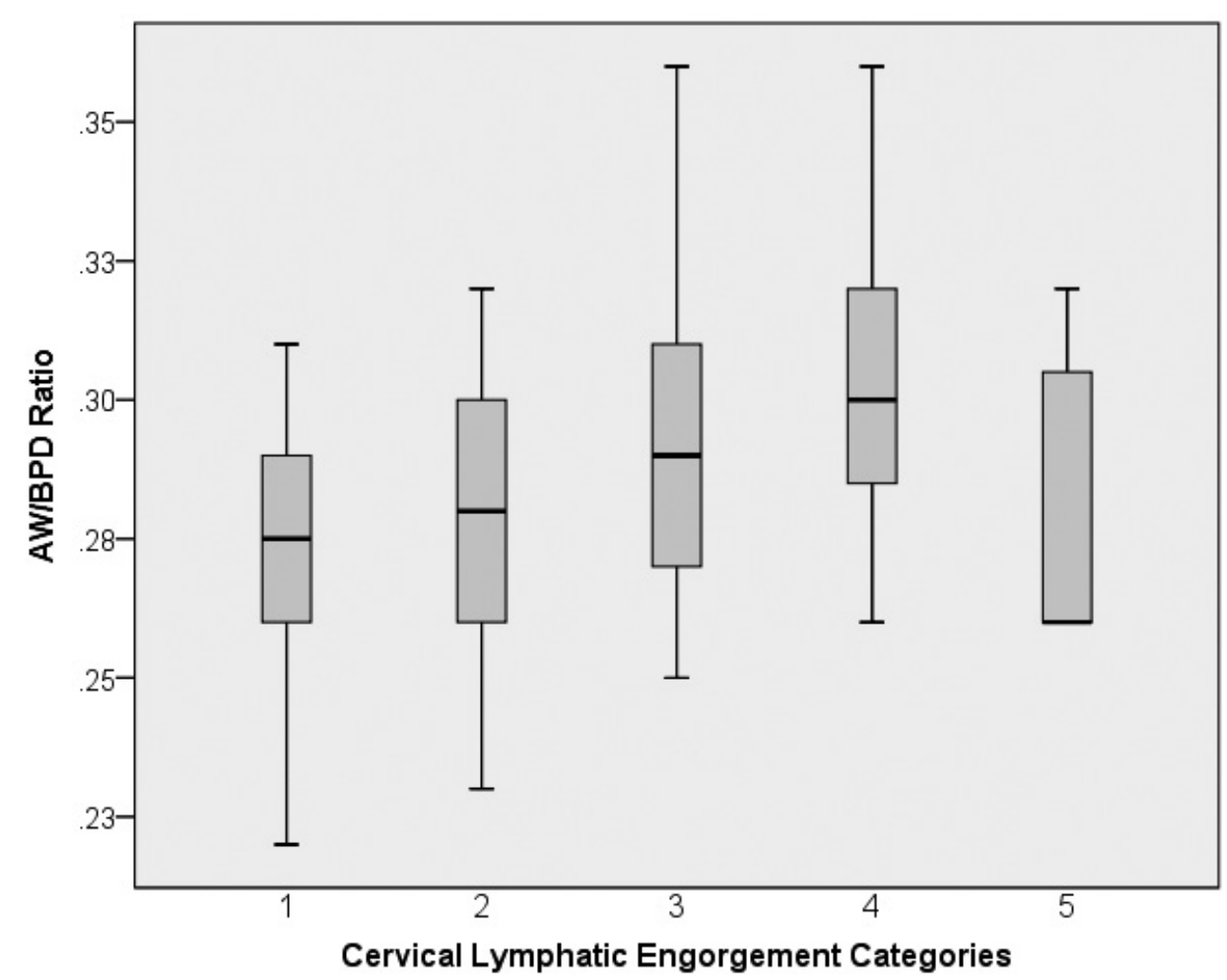

Fig. 1. Boxplot showing mean AWIBPD ratio in the different categories of cervical lymphatic engorgement (1: normal NT, no malformations; 2: enlarged non-septated NT 2.5-3.9 mm, no hydrops; 3: grossly enlarged non-septated NT / edema $>3.9 \mathrm{~mm}$; 4 : cystic hygroma and/or NIHF; 5: major anomalies with $\mathrm{NT}<2.5 \mathrm{~mm}$ ). Whisker bars indicate $95 \%$ confidence intervals.

position of the callipers on the corresponding coronal plane. Once the atrial width measurement was taken, the result was divided by the Biparietal Diameter measured on the same image, to normalize data by fetal size (Fig. 1). It should be underlined that the choroid plexuses and lateral ventricles at a higher level than that where the BPD is conventionally measured in the $1^{\text {st }}$ trimester; this results in BPD values in this study being significantly lower than those expected for the same gestational age. Cases with lymphatic engorgement signs were placed into 3 categories: NIHF and cystic hygroma, grossly enlarged NT or nuchal edema, and enlarged NT. NIHF was diagnosed if severe diffuse subcutaneous edema was associated with pleural effusion and/or ascites. Cystic hygroma was diagnosed if the fetus had a septated lymphatic nuchal enlargement, possibly extending caudalad to the trunk, but without ascites or pleural effusion. Grossly enlarged NT or edema was diagnosed if the retro-nuchal space was not clearly septated, measured $>3.9 \mathrm{~mm}$, and if pleural effusion and/or ascites were absent. Enlarged NT was defined by a nuchal translucency thickness of 2.5-3.9 mm, in absence of hydrops and septations. The occurrence of major congenital anomalies was irrelevant to this lymphatic system overload classification. However, cases of major anomalies not associated with nuchal enlargement were assessed separately. Therefore, this resulted in 5 categories: 1 ) normal NT, no malformations; 2) enlarged non-septated NT $2.5-3.9 \mathrm{~mm}$, no hydrops; 3) grossly enlarged non-septated NT 
/ edema $>3.9 \mathrm{~mm}$; 4) cystic hygroma and/or NIHF; and 5) major anomalies with NT $<2.5$ $\mathrm{mm}$. Presence of dilatation of the latero-cervical jugular lymphatic sacs was also recorded. The following variables were also recorded for all cases: presence of major congenital anomalies, karyotype, pregnancy and neonatal outcome. Karyotype was known in 82/96 $(85.4 \%)$ cases by means of prenatal invasive diagnosis (20 fetuses with aneuploidies and 41 euploid) or postnatal follow up (the remaining 21 euploid fetuses). Overall, there were 8 cases of trisomy 21,7 cases of trisomy 18,2 cases of unbalanced translocations and 1 case each of trisomy 13, monosomy X0, and mosaic tetra-12p (Pallister-Killian syndrome). Final outcome and confirmation of the malformations detected were available for the cases that reached the $2^{\text {nd }}$ trimester and/or were delivered at term. In the cases undergoing termination of pregnancy at the end of the $1^{\text {st }}$ trimester, the ultrasound diagnosis of the malformation was considered as confirmatory only if the anomaly was clearly evident beyond any reasonable doubt (e.g., omphalocele). Intra-operator and inter-operator variability was assessed on a random sample of 20 cases on which a repeated measurement was taken by one operator (DP) twice (intra-operator variability) and then by a second operator (GD; inter-operator variability).

Statistical analysis was performed with the SPSS software (SPSS, version 20.0). The One-way ANOVA test was used to compare means, with Tukey post-hoc test to evaluate inter-class differences. Intra- and inter-observer variability were assessed by means of the Cronbach' Alpha coefficient. P values $<0.05$ were considered statistically significant.

\section{RESULTS}

A summary of the 96 fetuses classified by cervical lymphatic engorgement categories and karyotype is shown in Table 1. In Fig. 1, the mean AW/BPD ratio by diagnostic category is shown. In particular, the AW/BPD ratio was significantly higher in fetuses with

\begin{tabular}{|c|c|c|}
\hline \multicolumn{3}{|c|}{$\begin{array}{c}\text { TABLE } 1 \\
\text { Summary of } 96 \text { Study Cases } \\
\text { Segregated by Cervical Lymphatic } \\
\text { Engorgement Category }\end{array}$} \\
\hline Category & Frequency (\%) & $\begin{array}{c}\text { Abnormal } \\
\text { karyotype (\%) }\end{array}$ \\
\hline $\mathbf{0}$ & $32(33.3)$ & 0 \\
\hline 1 & 19 (19.8) & $6(31.6)$ \\
\hline 2 & 22 (22.9) & $7(31.8)$ \\
\hline 3 & $15(15.6)$ & $5(33.3)$ \\
\hline 4 & $8(8.3)$ & $2(25.0)$ \\
\hline Total & $96(100)$ & 100.0 \\
\hline
\end{tabular}

0: Normal NT, no anomalies; 1: non-septated NT 2.5-3.9 mm, no hydrops; 2: non-septated NT (or edema) $>3.9 \mathrm{~mm}$, no hydrops; 3 : fetal hydrops or septated cystic hygroma; 4: major congenital anomalies, normal NT, no hydrops) and karyotype.

grossly enlarged NT/nuchal edema and NIHF/ septated cystic hygroma than in normal ( $p$ $<0.05$ and $\mathrm{p}<0.01$, respectively). Also, the AW/ $\mathrm{BPD}$ ratio was significantly higher in NIHF/ septated cystic hygroma than in enlarged NT 2.5-3.9 $\mathrm{mm}(\mathrm{p}<0.05)$. Figure 2 shows the case series stratified by presence or absence of dilated jugular lymphatic sacs (JLS). In category 2 (enlarged NT 2.5-3.9 mm), the AW/BPD ratio is significantly higher in presence of JLS $(\mathrm{p}<0.01)$. No relationship was found between abnormal karyotype and degree of cervical lymphatic engorgement. As for the inter- and intra-operator variability, this was fair, with Cronbach's alpha coefficients of 0.79 and 0.88 , respectively. Figure 3 demonstrates measurement of the AW/BPD ratio.

\section{DISCUSSION}

The hypothesis of this study is that in the fetus, as well as in the adult, there is a communication between the ventricular system and the cervical lymphatics via the cribriform plate. Due to the absence of lymphatics in the brain, the cerebrospinal and the interstitial fluids, together with the recently discovered glymphatic system (5), would act as a recently discovered lymph-like system conveying 


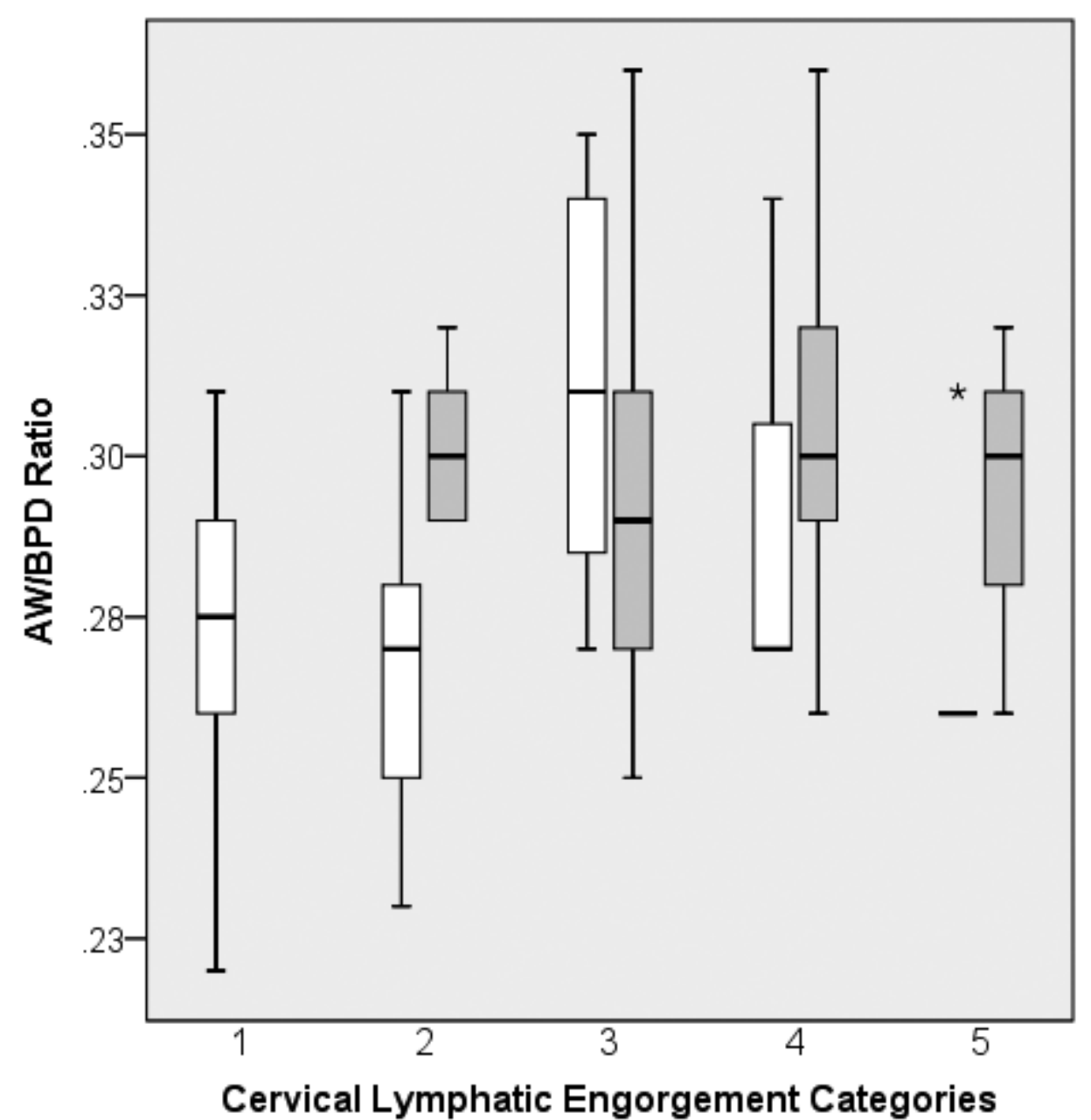

Fig. 2. Cluster boxplot showing mean AWIBPD ratio in the different categories stratified by presence or absence of jugular lymphatic sacs (JLS) dilatation (white bars: absence of JLS dilatation; grey bars: presence of JLS dilatation. Whisker bars indicate $95 \%$ confidence intervals. The asterisk indicates an outlier.

solutes and wastes from the brain to the cervical lymphatics. This mechanism is active alongside the historical one represented by reabsorption through the arachnoid granules of Pacchioni that are present as villi in the late third trimester, as granulations at $\mathbf{3 9}$ weeks, and become fully functional somewhat later (6). Therefore, it may be speculated that in prenatal life the system responsible for clearing catabolites and wastes from the brain is relying even more heavily on the aforementioned path, i.e., active trans-membrane and paravascular passive flow across the crib- riform plate (5). No matter what is the real mechanism of this exit route from the brain, if there is an abnormal or delayed development of fetal jugular lymphatics, as in cystic hygroma or enlarged NT (7), then this should theoretically reduce the actual speed of the whole process, with a consequent engorgement of the ventricular system? However, as pointed out $(2,8-11)$, this is only one of the routes through which it has been speculated that waste and solutes exit from the brain in the absence of a local lymphatic network (5). This is why what we expect is only a moderate overload of the 

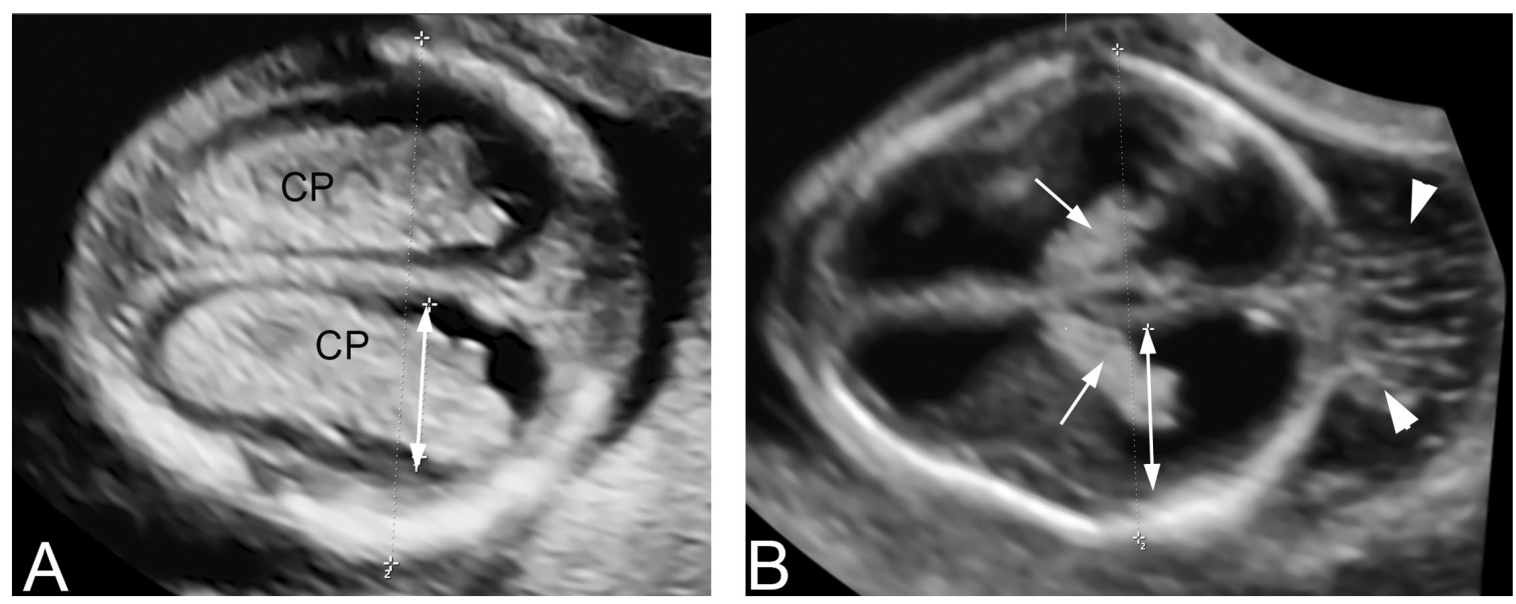

Fig. 3. Measurement of the AWIBPD ratio. The width of the atrium (double arrow) is measured in between the atrial walls, if visible; if the walls are adjacent to the choroid plexus (as in A), the calipers are placed just external to the choroid plexus. A) Normal fetus, 13 gestational weeks. The image shows the modified axial view of the fetal head on which the AWIBPD ratio is measured. Note the large choroid plexuses $(C P)$, normal for this gestational age, which fill most of the lateral ventricle, and the midline. B) Fetus with hydrops (note the severe nuchal edema, arrowheads). Note how the ventricle appears prominent due to the compression of the choroid plexuses from the increased amount of cerebrospinal fluid in the ventricles.

ventricular fetal system. In this study, we have addressed this very point, i.e., whether early cervical lymphatic obstruction is associated with a sonographically detectable dilatation of the ventricular system in the $1^{\text {st }}$ trimester of pregnancy. Our preliminary results seem to support this concept; fetuses with septated cystic hygroma and/or hydrops and those with grossly enlarged non-septated NT $(>3.9 \mathrm{~mm})$ or nuchal edema show a significantly higher AW/BPD ratio than normal fetuses (Fig. 2). By looking at Fig. 1, it can be visually appreciated that the more pronounced the cervical lymphatic engorgement is, the higher the degree of ventricular system dilatation, even though there is only a significant difference between categories 3 and 4 and category 1 ( $p<0.05$ and $\mathrm{p}<\mathbf{0 . 0 1}$, respectively). An interesting finding is that in fetuses with moderately enlarged NT $(2.5-3.9 \mathrm{~mm})$ the degree of ventricular system overload is significantly higher in case of JLS dilatation (Fig. 3; p <0.01). Since it is known that, in some cases, severely enlarged NT shrink due to drainage into the JLS, it can then be speculated that those cases with moderately enlarged NT and dilated JLS have a higher degree of cervical lymphatic blockage than fetuses with similar NTs but no JLS. And, if our hypothesis is true, in the latter instance a higher degree of ventricular system dilatation would be expected, as it is indeed the case (Fig. 2). An expected query on the results of this preliminary study is that if indeed a cervical lymphatic engorgement would trap cerebrospinal fluid inside the brain, then the expected degree of the ventricular system dilatation should be more pronounced than that observed in this investigation. A possible answer to this point is that probably there is more than just one system in place to ensure proper clearance of waste and solute from a metabolically very active and precious organ such as the brain, even in the absence of the Pacchioni granules, which could be the case for the fetus. The problem is that science has not yet fully unveiled these mechanisms $(2,5)$.

This study has some limitations. First, the number of cases is relatively limited, but we preferred to exclude cases in which an adequate three-dimensional volume dataset was not available in order to increase the reproducibility of the measurement. This led to a signif- 
icant number of potential cases being excluded from the analysis because most volumes had been acquired starting from the midsagittal view needed for the NT assessment. Due to the ill-alignment, this resulted in an inadequate visualization of the ependymal edges of the lateral ventricles. Second, in some cases the choroid plexuses completely filled the ventricles, and this masked the actual ependymal walls. In these cases, we assumed that the edges of the choroid plexuses corresponded to the actual walls of the ventricles and we double-checked the measurement by looking at the corresponding coronal view using the multiplanar image correlation advantage provided by the $3 \mathrm{D}$.

In conclusion, this preliminary investigation seems to confirm that at the end of the first trimester, presence of cervical lymphatic engorgement, in terms of grossly enlarged NT, nuchal edema, septated cystic hygroma and NIHF, is statistically associated with a moderate dilatation of the ventricular system. Of note, among fetuses with moderately enlarged NT, those with evidence of dilatation of the JLS, which represent an abnormal or delayed development of the cervical lymphatic network, show a statistically significant increase in the AW/BPD ratio. However, further studies are needed, both in the experimental animal setting and in humans, to increase the currently patchy knowledge of the actual relationships among cerebrospinal fluid, cervical lymphatic drainage, and NIHF.

\section{ACKNOWLEDGMENT}

This research was supported by the Eu-Brain Non-Profit Association (LAR).

\section{CONFLICT OF INTEREST AND DISCLOSURE}

All authors declare that no competing financial interests exist.

\section{REFERENCES}

1. Bellini, C, G Donarini, D Paladini, et al: Etiology of non-immune hydrops fetalis: An update. Am. J. Med. Genet. A(5) (2015), 1082-1088.

2. Bellini, C, RC Hennekam: Non-immune hydrops fetalis: A short review of etiology and pathophysiology. Am. J. Med. Genet. Part A. 158A (2012), 597-605.

3. Begley, DJ: Brain superhighways. Sci. Transl. Med. 4 (2012), $147 \mathrm{fs} 29$.

4. Nicolaides, KH: Screening for fetal aneuploidies at 11 to 13 weeks. Prenat. Diagn. 31 (2011), 7-15.

5. Iliff, JJ, M Wang, Y Liao, et al: A paravascular pathway facilitates CSF flow through the brain parenchyma and the clearance of interstitial solutes, including amyloid B. Sci. Transl. Med. 4 (2012), 147 ra111.

6. Papaiconomou, C, R Bozanovic-Sosic, A Zakharov, et al: Does neonatal cerebrospinal fluid absorption occur via arachnoid projections or extracranial lymphatics? Am. J. Physiol. Regul. Integr. Comp. Physiol. 238 (2002), R869-876.

7. Bellini, C, M Rutigliani, FM Boccardo, et al: Nuchal translucency and lymphatic system maldevelopment. J. Perinat. Med. 37 (2009), 673-676. doi: 10.1515/JPM.2009.107.

8. Bellini, C, F Boccardo, E Bonioli, et al: Lymphodynamics in the fetus and newborn. Lymphology 39 (2006), 110-117.

9. Johnston, M, C Papaiconomou: Cerebrospinal fluid transport: A lymphatic perspective. News Physiol. Sci. 17 (2002), 227-230

10. Hladky, SB, MA Barrand: Mechanisms of fluid movement into, through and out of the brain: evaluation of the evidence. Fluids Barriers CNS11 (2014), 26.

11. Louveau, A, I Smirnov, TJ Keyes, et al: Structural and functional features of central nervous system lymphatic vessels. Nature 523 (2015), 337-341.

Carlo Bellini, MD, PhD

Neonatal Intensive Care Unit, Department of Mother \& Child

IRCCS Gaslini

Largo G. Gaslini, 5

16147 Genoa, Italy

Tel: 0039-010-56362762

Email: carlobellini@gaslini.org 